Hauke Brunkhorst

\title{
Neustart - Kritische Theorie Internationaler Beziehungen
}

Während das realistische Paradigma zu abstrakt ist, um eine komplexe gesellschaftliche Realität zu begreifen, in der das Normative (einschließlich Ideen, Kultur usw.) eine von Macht und Kapital verschiedene Realität in der Realität darstellt, ist das normative Paradigma zu abstrakt um die Realität einer unkontrollierbaren Evolution von Kapital, Macht usw. noch zu erreichen. Stattdessen wird ein drittes Paradigma vorgeschlagen, das im Rückgang zu den holistischen Gesellschaftstheorien gewonnen wird. Es wird gezeigt, wie man sich seine Leistung als kritische Theorie der IPT vorstellen könnte.

\section{Vorbemerkung}

Als Soziologe bin ich nicht vom Fach und habe nur eine grobe Übersicht über das in der Internationalen Politischen Theorie (IPT) vorhandene Theorieangebot. Wenn ich mir aus dieser beschränkten und externen Perspektive überhaupt ein Urteil erlauben darf, lässt sich das vorhandene Angebot sehr grob so sortieren, dass sich realistische und normativistische Theorieoptionen gegenüberstehen (Abschnitt 2). Zunächst argumentiere ich, dass schon die - zu Unrecht vergessene und verdrängte - holistische Gesellschaftstheorie Talcott Parsons (Abschnitt 2.2) komplex genug gebaut ist, um die komplementären Schwäche realistischer (Abschnitte 2.1 und 2.3) und normativistischer Positionen (Abschnitt 2.4) aufzuheben. Im Folgenden schlage ich dann einen Rückgriff auf die Theoriebaukästen der holistischen Gesellschaftstheorien, der Systemtheorie und der kritischen Theorie vor, um zunächst knapp für eine Ergänzung des Begriffs der Internationalen Beziehungen (IB) um den der Internationalen Gesellschaft bzw. Gemeinschaft (IG) zu plädieren (Abschnitt 3.1) und sodann eine perspektivische Einbeziehung der Weltgesellschaft und des Internationalen Rechts vorzuschlagen, die es erlaubt, die »Vernunft des Vergleichs« (Luhmann 1965: 8, 202-203) für die Theoriebildung zu nutzen (Abschnitt 3.2). Zu diesem Zweck bietet es sich an, den affirmativen Normativismus des Neo-Kosmopolitismus durch eine negativistisch begründete, reflexive Gesellschaftstheorie abzulösen, die Normativismus und Realismus vermittelt (Abschnitt 3.3). Ein brauchbares Paradigma solcher Theoriebildung ist immer noch »Das Kapital« von Karl Marx (1867). Es wäre völlig verfehlt, das Werk - wie es heute gelegentlich wieder geschieht - als wirtschaftspolitisches Hilfsprogramm für die ins Schlingern geratene Globalisierung zu lesen. Es stellt vielmehr das Modell einer holistischen Gesellschaftstheorie dar, das Klassenkampf (sozialen Konflikt) und Evolution, normative Lernprozesse und sozialstrukturelle Selektion (Anpassung) exemplarisch verschränkt. Um daraus noch Nutzen zu 
ziehen, muss es freilich hoch generalisiert und einem sehr viel abstrakteren Theoriedesign eingepasst werden (Abschnitt 4).

\section{Geistloser Realismus und leeres Sollen}

\subsection{Kategoriale Verleugnung}

Realistische Theorien, die bis auf Hegels Rechtsphilosophie und die ganze dahinter stehende Tradition politischer Ideen seit Hobbes und Macchiavelli ${ }^{1}$ zurückgehen, waren in der Zeit zwischen den beiden Weltkriegen bis Ende der 1940er Jahre heftig umstritten $^{2}$, dann, während des sogenannten Kalten Kriegs, gewann die realistische Theorie eine hegemoniale Stellung ${ }^{3}$, die - trotz heftiger Gegenbewegungen in den 1960er Jahren und um die Jahrhundertwende - bis heute ungebrochen zu sein scheint. Aber Erfolg ist nicht Wahrheit, und die letale Schwäche der sich selbst realistisch nennenden Schulen liegt in der notorischen Unterschätzung und kategorialen Verleugnung der faktischen Kraft des Normativen. Keine Gesellschaft kann ohne normative Integration überleben. Der Einwand, den Talcott Parsons schon 1961 den damals dominanten realistischen Theorien internationaler Beziehungen entgegenhielt, trifft auch heute noch den Nagel auf den Kopf (Parsons 1961).

\subsection{Return of Parsons}

Alle Institutionen, auch die der internationalen Beziehungen, sind $»$ Formen generalisierter Verhaltenserwartungen « (Luhmann 1965: 13). Sie stellen jeweils eine normative Ordnung von Interessen dar, die als solche einen Verpflichtungscharakter hat, der sich nicht auf die durch sie geordneten Interessen reduzieren lässt, sondern als normativ richtige Ordnung anerkannt werden muss, um Bestand zu haben (Parsons 1961: 121, 125). In dem (fiktiven Hobbesschen) Augenblick, in dem das Selbstinteresse sich zu einer normativen Ordnung formt, »self-interest comes to be bound up with conformity with a normative order « (Parsons 1961: 124). Letztere bringt ein jeweils allgemeines Interesse zur Geltung: Geraten die »requirements« dieser »normative order [...] in serious conflict with the self-interests of all the units related to it, [it] could not be upheld for very long« (Parsons 1961: 124, meine Hervorh.). Schon

1 Wie man freilich Macchiavelli »gegen den Strich bürsten« (Benjamin 1974: 253) und in einen »red republicanism« verwandeln kann, zeigt Banu Bargu (2010).

2 Siehe nur die staats- und verfassungsrechtlichen Kontroversen der Weimarer Juristen und Völkerrechtler, in den konträren Positionen einerseits Hans Kelsen (1960), andererseits Carl Schmitt (1933); aber auch Hermann Heller (1971); zur Entwicklung während des Ersten Weltkriegs Eberl (2007); wichtige Sammelbände zur Weimarer Debatte: Gusy (2000); Staff/ Müller (1985); Waschkuhn/Thumfart (2002); Gangl (2001; 2008); Brunkhorst/Voigt (2008); im gesellschaftlich-geschichtlichen Kontext: Brunkhorst (2008).

3 Vgl. Morgenthau (1948); Rosenthal (1991); Herz (1951); vgl. zum ideengeschichtlichen Kontext: Koskenniemi (2002). 
aus der Tatsache, dass die moderne »international order« eine komplexe Gesellschaftsordnung, ein hoch differenziertes »social system « ist, das aus Staaten, grenzüberschreitenden privaten Assoziationen, globalen Handelsbeziehungen, private public partnerships (die ersten waren die calvinistisch geprägten East India Companies Englands und der Niederlande im 17. Jahrhundert) ${ }^{4}$, internationalen Organisationen, Rechtsordnungen und Gerichten, Institutionen und Foren der Weltöffentlichkeit (United Nations General Assembly usw.) besteht, folgt, dass die »components of order«, also z. B. die lex mercatoria, aber auch die staatsbezogenen (Aggressionsverbot, Genozidkonvention etc.) und individualrechtlichen (Hospitalität, internationale Menschenrechte etc., vgl. schon Kant 1977: 213) Normen des internationalen Rechts keine »simple function of the political policies of government « (Parsons 1961: 123-126) sein können. Das zu behaupten, ist empirisch einfach falsch. Die faktische Stabilität internationaler Beziehungen kann deshalb nicht, wie die realistischen Schulen annehmen, allein und noch nicht einmal vorrangig auf »'power' relations « gegründet sein. Schon das globale politische System ist für sich betrachtet nicht nur durch Macht und Machtbalancen, sondern ebenso sehr durch ökonomische Produktivität, durch eine autonome normative Ordnung und durch kulturelle Wertbindungen konstituiert, erst recht gilt das für die globale Gesellschaftsordnung, das »social system« der »entire world«(Parsons 1961: 120). Wie Parsons (1961: 126-128) dann am Weltsystem des sogenannten Kalten Kriegs einleuchtend zeigt, muss dieses System als eine (wie immer defizitäre) »'constitutional' order « beschrieben werden (dazu sogleich mehr) und nicht, wie in den realistischen Schulen, als Fortsetzung des Kriegs mit den andern Mitteln politischer Macht.

Lässt man das in den IB nach wie vor hegemoniale Hobbessche, durch John Rawls (1971) vergeblich wiederbelebte Modell des Naturzustands (Anarchie internationaler Beziehungen) auch als heuristische Fiktion - die es nie nur war, enthielt es doch immer die autoritäre Warnung vor dem Rückfall in den wirklichen Naturzustand gänzlich fallen, so wird man mit der Phänomenologie von der schlicht realistischen und empirisch unwiderlegten Annahme ausgehen müssen, dass interagierende menschliche Individuen, soziale Systeme und Organisationen immer schon normativ integriert sind (zur Kritik der Anarchie-Prämisse vgl. Kessler 2010, in diesem Heft). Nicht erst die instrumentelle Lösung des »Hobbesian problem of order« (Parsons

4 Eine (Rechts-)Geschichte der private-public partnerships seit Beginn des modernen Staats im 16./ 17. Jahrhundert gibt es, wenn ich es recht sehe, bislang noch nicht. Sie könnte $\mathrm{zu}$ weiteren erheblichen Revisionen am nach wie vor hegemonialen Bild des Anstaltsstaats nötigen. Dieses Bild ist zwar mittlerweile durch Berge widerspenstiger Tatsachen und sich abzeichnende, alternative Paradigmen eingetrübt, wird aber immer noch durch das Frontispiz des Hobbesschen Leviathan verzaubert. Unter diesem Aspekt könnte man vielleicht auch Horst Bredekamps (1999) bedeutende Studie nochmals gegen den Strich bürsten; vgl. auch Brandt (1982). Das einseitige Bild des Leviathan ist immerhin schon durch die älteren, republikanischen Gegenbilder relativiert worden; vgl. Hoffmann (1977); Skinner (1989). Mir sind (ich kenne mich hier aber nicht aus) keine systematischen Studien bekannt, die den visuellen Gegenstrategien zum herrschenden Bild des Leviathan nachgingen. Sie wären aber - insbesondere für die junge Disziplin der Internationalen Politischen Theorie - von größtem Interesse, sowohl die republikanischen und basisdemokratischen wie die kosmopolitischen Gegen-Bilder. 
1937: 91) war der Fehler, schon das Problem selbst ist ein klassisches Scheinproblem, das sich der falsch gestellten Frage verdankt, wie man aus einem Naturzustand herauskomme, in dem sich niemand nie befunden hat. Deshalb haben sich auch die Staaten nie im Naturzustand befunden, weil irgendeine Form normativer Integration in dem Augenblick unvermeidlich wird, indem sie beginnen, miteinander zu kommunizieren. ${ }^{5}$ Genau an dieser Stelle werden die soziologischen Studien der Stanford School um John W. Meyer $(1997 ; 2005)$ für die IB und die IPT interessant, verbinden sie doch die systemtheoretische Einsicht in die evolutionäre Existenz einer singulären Weltgesellschaft mit der dann naheliegenden, makrophänomenologischen Vermutung eines für diese Gesellschaft im Ganzen konstitutiven kulturellen Wissens, das auch normative und evaluative Grundorientierungen einschließt. ${ }^{6}$ Die Makrophänomenologie soll nicht (mikrophänomenologisch) erklären, was sowieso jeder weiß, dass die Welt überall und immer wieder anders aussieht und die Kulturen dieser Welt höchst vielfältig und an jeder Straßenecke andere Deutungsmuster hervorbringen, sondern warum so viele Dinge überall auf der Welt so gleich oder einander ähnlich sind und dieselbe Struktur aufweisen (Regierungen, politische Rhetorik, Erziehungsziele, Bildungssysteme, Wertorientierungen, Organisationen usw.).

\subsection{Theoriedesign}

Aber schon die Argumente von Parsons konnten die Realisten 1961 nur kopfschüttelnd zur Kenntnis nehmen und unter Ideologie verbuchen. Doch nicht Parsons, sie waren die Ideologen. Selbst wenn sie den Mangel schließlich spürten, hatten sie kategoriale Schwierigkeiten, normative Gesichtspunkte systematisch in Rechnung zu stellen (Scheuerman 2009). Das Paradigma der Souveränität, juristisch leere Begriffe wie Kompetenz-Kompetenz, der Vorrang des nationalen Selbsterhaltungsinteresses, der methodische Nationalismus (Beck o. J.), der strategische Reduktionismus in der Handlungstheorie, der mit Voluntarismus und Irrationalismus psychologisch kompensiert, aber nicht aufgehoben werden kann, und der buchstäblich ideenlose Empirismus fungieren hier als transzendentales Verdrängungsschema. Normen werden entweder dem Wolkenkuckucksheim des Wünsch-, aber kaum Realisierbaren zugeschlagen (wie die Welt sein sollte) oder (in der Welt) als ideologische Tarnung instrumenteller Interessen missverstanden. Die deshalb nur vermeintlich realistische Beobachterperspektive ist durch diese kategoriale Engführung entweder (von Carl Schmitt bis Samuel Huntington, von Hans Morgenthau bis Kenneth Waltz) auf die

5 Das gilt selbst für die schlimmsten kriegführenden Staaten der Geschichte. Sonst hätte es ja auch nicht den geringsten Sinn gemacht, jemals nach der Berechtigung von und der Schuld an Kriegen zu fragen.

6 Dazu auch Brunkhorst (2009). Zu Recht verweist auch Antje Wiener (2010, in diesem Heft) auf die Bedeutung von Meyer und der Stanford School für die Entwicklung eines politikwissenschaftlichen Begriffs von »global constitutionalism«. 
Autonomie des Politischen (bzw. des politischen Systems ${ }^{7}$ ) oder (in orthodox marxistischen Theorien des Staats wie bei Perry Anderson oder in der marxistisch inspirierten Weltsystemtheorie Immanuel Wallersteins) auf die Autonomie des Ökonomischen fixiert worden. ${ }^{8}$ Während die einen die Autonomie der Macht und die Heteronomie des Kapitals behaupten, behaupten die andern die Autonomie des Kapitals und die Heteronomie der Macht. Was den einen die Geopolitik, ist den andern der Imperialismus, aber immer ist die Prämisse, dass es nur ein autonomes Führungssystem geben kann und Führung sich auf die Wahrung und Durchsetzung des jeweils strategischen Selbstinteresses reduziert. Dagegen argumentiert Parsons realistisch mit dem Argument, dass Führung nicht mit Macht verwechselt werden dürfe:

»The alternative interpretation is that not only do the positions of the United States and the Soviet Union [im Jahr 1961] respectively contain elements of sheer power - which of course are there - but that they also contain elements of genuine leadership. Leadership in this sense exists only when there is a political support for the position, backed by interests other than the most elementary security and subject to an accepted (i. e. institutionalized) normative order« (Parsons 1961: 125, meine Hervorh.).

Und eine solche Ordnung übergreift auch noch die Gegensätze der Konfliktparteien des Weltsystems von 1961, das Parsons deshalb als constitutional order mit einem »internal partisan conflict« (wie dem zwischen konservativen und progressiven Parteinen in parlamentarischen Regierungssystemen) beschreiben kann, der im Medium internationalen öffentlichen Rechts und einer rechtlich institutionalisierten Weltöffentlichkeit (»world opinion«) ausgetragen wird (Parsons 1961: 125).

Das Theoriedesign der realistischen Schulen ist hoffnungslos unterkomplex. Schon die gleichzeitige Autonomie verschiedener Systeme und die Gleichzeitigkeit von (absoluter) Unabhängigkeit (Autopoiesis/Selbstproduktion) und vollständiger Umweltabhängigkeit können sie kaum angemessen konzeptualisieren. In dem vielleicht produktivsten dieser Forschungsprogramme, der Staatsformationstheorie Charles Tillys (1990), wird dieser Mangel zwar dadurch behoben, dass Kapital (Wirtschaft) und Zwangsmacht (Politik) jederzeit gleichermaßen autonome Organisationsformen finden können (z. B. über Jahrhunderte Territorialstaaten und Städte) und damit die Frage des Vorrangs oder Gleichgewichts zwischen Politik und Wirtschaft zu einer rein empirischen Frage wird, die je nach Geschichtsverlauf mal so, mal anders beantwortet werden muss (ähnlich ten Brink 2008). Aber auch Tilly ist für die Autonomie des Rechts, die für die evolutionäre Dynamik gerade der modernen Gesellschaft von entscheidender Bedeutung ist, erstaunlich blind, und auch in dieser realistischen Variante politischer Gesellschaftsgeschichte bleibt das dialektische Wechselspiel von Ideen und Interessen bzw. von ideellen und materiellen Interessen (im Sinne von Max

7 Denen, die hier eine ontologische Differenz zwischen dem Politischen und dem politischen Handeln bzw. dem politischen System postulieren, kann man nur mit Theodor Adorno antworten, kein Sein ohne Seiendes, also: kein Politisches ohne Politik.

8 Knappe und treffende Kritik an beiden Positionen in Tilly 1990. 
Weber) stark unterbelichtet. ${ }^{9}$ Auch hier scheitert der instrumentelle Realismus letztlich an der sozialen Realität des Normativen.

\subsection{Undialektischer Normativismus}

Wenn die Welt sich verändert und strukturelle Barrieren fallen, kommt die Stunde des Sollens und der utopischen Entwürfe. Das ist vielleicht das einzige, was die Jahreszahlen 1789 und 1989 miteinander verbindet. Die Implosion des riesigen Sowjetreichs hat das letzte, am Ende nur noch durch Zwang zusammengehaltene TerritorialImperium klassischen Zuschnitts zum Verschwinden gebracht, der gegen 1789 und 1918 gerichteten, neokonservativen Konterrevolution neuen Auftrieb gegeben und den gesamten Globus in ein einziges politisches System von Nationalstaaten und Welt- und Regionalorganisationen verwandelt, das die Schleusen für die vollständige Globalisierung der Weltöffentlichkeit und aller Funktionssysteme und kulturellen Wertsphären weit geöffnet hat. Das hat die normativistischen Theorien aus der Reserve gelockt, so dass sie zumindest eine Zeitlang den hegemonialen Realismus in die Defensive treiben konnten. ${ }^{10}$ Starke theoretische Impulse sind dabei von den Arbeiten von Rawls (1999) und Jürgen Habermas (2004) zum law of the peoples und zur Konstitutionalisierung des Völkerrechts ausgegangen. Das hat einerseits zu einer neuen Blüte des Kosmopolitismus geführt (Neo-Kosmopolitismus) ${ }^{11}$, andererseits die ihrerseits normativ motivierte Kritik poststrukturalistischer, dekonstruktiver, postkolonialer und postmarxistischer Strömungen herausgefordert (hierzu ausgewogen: Brunkhorst 2010a). Zumindest teilweise ist diese Kritik berechtigt, was sich äußerlich schon daran zeigt, dass ein repräsentativer englischer Band zum Thema internationaler Beziehungen, der »Fifty key thinkers« (Griffiths et al. 2009) des Faches vorstellt, das Kapitel über »International Political Theory« gleich auf »International Ethics« reduziert und auch die dazu passende einseitige Auswahl wichtiger Denker trifft. Aber schon im Begriff der Theorie, darauf weist Peter Niesen (2010) zu Recht hin, schließt die IPT weit mehr als rein ethische und normative Fragen ein, auch wenn die faktische Theoriebildung bisweilen hinter ihrem eigenen Begriff zurückbleibt.

9 Das gilt ebenso für die interessante neo-post-neo-marxistische Studie von ten Brink (2008). Zu Recht wird hier das seit den 1980er Jahren verdrängte Erklärungspotential marxistischer Theoriekonzepte reaktualisiert, aber der für den Juristen Marx zentrale Begriff des Klassenkampfes bleibt weitgehend ausgeblendet und, wie es scheint, nicht nur aus thematischen Gründen. Klassenkämpfe sind aber das eigentliche Medium von normativen Lernprozessen, in denen sich nicht nur materielle, sondern auch ideelle Interessen bilden: »Der Mensch revoltiert nicht fürs Brot allein« (Moore 2001: 169). Vgl. auch Eder (1983) und Moore (1978).

10 Vgl. den knappen Überblick von Niesen (2010, in diesem Heft), der insgesamt diesen Trend bestätigt. Ich würde jedoch im Unterschied zu Niesen den Unterschied zwischen IPT als Internationale Ethik (in der Nachfolge von Hugo Grotius, Samuel von Pufendorf, Emmerich de Vattel) und IPT als Theorie legitimer internationaler Institutionen (in der Nachfolge Kants) nicht überbewerten und beide als, freilich sehr verschiedene Varianten normativer Theorie verstehen.

11 Gute und knappe Übersicht bei Kleingeld (2006); vgl. auch Held (1995); Höffe (1999); Gosepath/Merle (2002). 
Viele normativistische Neuansätze kosmopolitischer Theorien, ob sie sich nun der IPT, der politischen Philosophie, den IB oder der politischen Theorie zuordnen, lassen jedoch nicht nur jeden Sinn für die vertrackte Dialektik der Aufklärung vermissen, auf sie trifft auch der etwas abgedroschene, seinerzeit zu Unrecht auf Immanuel Kant gemünzte Vorwurf Georg Wilhelm Friedrich Hegels zu (vgl. Schnädelbach 2000: 65-67), es handle sich bei ihnen um nichts als leeres Sollen. Am grünen Tisch normativer Pläne erscheint die objektive Undurchdringlichkeit systemischer Komplexität ebenso wie die interessengeleitete Widerständigkeit struktureller Herrschafts- und Klassenverhältnisse dann bestenfalls als restriktive Randbedingung politischen Handelns, die dessen reine Vernunft, wenn es nur durch die richtige Theorie der Gerechtigkeit angeleitet ist, nicht zu trüben vermag. In der Subdisziplin der Internationalen Beziehungen war Herrschaft »lange Zeit« ein »kaum genutztes Konzept «. ${ }^{12}$ So blind wie Interessen ohne Ideen sind, so leer sind Ideen ohne Interessen. Zwar erinnern die normativistischen Theorien mit großem Recht an die im Realismus verdrängte, weichenstellende Rolle von Ideen in der sozialen Evolution, aber sie versuchen oft gar nicht erst, ihre Theorie der Gerechtigkeit zumindest auch als Evolutionstheorie und in deren Begrifflichkeit zu konzeptualisiseren. ${ }^{13}$ Deshalb wird schon der eigentümliche Doppelcharakter des Rechts, seine Stellung zwischen Freiheit und Zwang, zwischen Funktion und Normativität, die Hegel auf den Begriff des objektiven Geistes gebracht hat, in der Regel zugunsten von Freiheit und Normativität vereinseitigt, was sich meines Erachtens an der normativen Überlastung des Menschenrechtsdiskurses in der politischen Philosophie und Theorie gut nachweisen ließe. ${ }^{14}$ Nur wenn es ihr gelingt, dieses Einfallstor für nur allzu berechtigte Ideologiekritik zu schließen, kann die normative Theorie progressive Forschungsprogramme (Lakatos 1974) entwickeln, um die Hegemonie des vermeintlichen Realismus von innen zu zersetzen.

12 Deitelhoff (2010: 285, in diesem Heft). Dort auch ein erster programmatischer Ansatz, den Herrschaftsbegriff im internationalen und globalen Kontext neu zu thematisieren. Dazu freilich genügt es nicht, die Habermassche Diskurstheorie in ein behavioristisches Forschungsprogramm umzuwandeln, das auf argumentatives Verhalten im Nahbereich programmiert ist (z. B. Thomas Risse und die Berliner Schule, z. T. auch Harald Müller und die Frankfurter Friedensforschung). Dazu kritisch: Wiener (2010); Kessler (2010) sowie Herborth/Niesen (2007).

13 Anders freilich: Habermas (1976a: 9, 63, 129, 144; 1981, 118, 229, 591; 1999: 7, 271). Aber auch Tugendhat (2001: 199). Schon Kants Philosophie, die ja noch am Beginn des evolutionistischen Denkens, das Mitte des 18. Jahrhunderts beginnt, steht, lässt sich retrospektiv, wie Vorländer (1921: 100) knapp zeigt, als »Verbindung« eines »darwinistischen mit einem teleologischen Gedanken« interpretieren.

14 Siehe nur die kritischen Studien von Kreide (2008) und Prien (2010). Siehe auch die Kritik von Rainer Forst (2010: 361, in diesem Heft) am »abstrakten Kosmopolitismus « und der »abstrakten Idealtheorie«, die von der »konkreten Wirklichkeit sozialer Verhältnisse der Beherrschung und Ausbeutung« abstrahiere. 


\section{Holistische Gesellschaftstheorie}

\subsection{Von IB zu IG}

Auch wenn es keinen Zweck hat, in ein konservatives Lamento über disziplinäre und disziplinierende Spezialisierung zu verfallen, und zwanghafte Interdisziplinarität die Wissenschaft auf das Niveau von Zwangsheiraten zurückbeordert, der zum Namen und Kürzel der Disziplin gewordene Begriff der Internationalen Beziehungen (IB) spiegelt lediglich die Hegemonie des Realismus und ist zu Recht um den Begriff der internationalen Gesellschaft bzw. Gemeinschaft (IG) ergänzt und erweitert worden. Der Begriff der IG (Mosler 1980, Tomuschat 1995) ist normativ auf eine Weltbürgergenossenschaft zugeschnitten, die im Kommen (Jacques Derrida) oder-als Begriff des positiven Völkerrechts - in the making ist. Mit dieser Erweiterung kommt nicht nur die Konkurrenz verschiedener Paradigmen auch begrifflich zur Geltung, sie setzt auch die Internationalen Beziehungen und das Internationale Recht (IR) in ein produktives Ergänzungsverhältnis. So erweist sich der Begriff der global society bzw. der globalen Gesellschaft (GG) als IB-Komplementärbegriff zum IR-Begriff der IG (vgl. Wiener 2010, mit Bezug auf Barnett/Sikkink 2008). Sowohl im Völkerrecht wie in den IB wird mit dieser Umstellung von Beziehung auf Gesellschaft/Gemeinschaft zunächst ein individualistisches Verständnis von Gesellschaft entwickelt, durch das Individuen als Akteure auf der Ebene der Weltgesellschaft (neben Staaten, internationalen Organisationen usw.) normativ oder faktisch ernst genommen werden (Wiener 2010). Dieser Individualismus stellt eine erneute Schranke beider Paradigmen (GG-IB und IG-IR) dar, die erst mit dem Begriff der Weltgesellschaft überwunden wird.

\subsection{Vernunft des Vergleichs}

Methodisch ist die Einengung auf politische Theorie nicht unproblematisch, zumal wenn es dann auch noch gleich zur Spezialisierung auf Internationale Politische Theorie kommt. Eine erneute und vor allem erweiterte Öffnung der disziplinären Grenzen zu den soziologischen Theorien der Weltgesellschaft wäre ebenso wichtig wie die Wiederaufnahme der auseinandergelaufenen Beziehung zum Völker- und Verfassungsrecht. ${ }^{15}$ Es käme dabei weniger auf disziplinäre Integration als auf die perspektivische Nutzung von Vergleichsmöglichkeiten an. ${ }^{16}$ Das erfordert jedoch den

15 Ich stimme hier ganz mit den Schlussfolgerungen aus Niesens Analyse (2010: 274) überein, der ebenso wie ich den Mangel eines Großteils normativer Theoriebildung in der fehlenden Analyse von weltgesellschaftlichen $\gg$ Verwirklichungsbedingungen präskriptiver politisch-theoretischer Aussagen« und einer weltgesellschaftlich ansetzenden Theorie »legitimen Institutionenwandels« sieht (zu letzterer mein Vorschlag unter 4.1 und 4.2).

16 In ähnliche Richtung gehen die Überlegungen von Kessler (2010) und Deitelhoff (2010). $\mathrm{Zu}$ Recht wehrt Deitelhoff (2010: 288) sich gegen »hegemoniale Grenzziehungen« der Disziplin, die »gesellschaftstheoretische Fragen [...] von vornherein als irrelevant abtun«. 
Rückgriff auf holistische Gesellschaftstheorien, die nicht nur komplex genug gebaut sind, um die wichtigsten Einsichten normativistischer und realistischer Ansätze zu vermitteln, sondern auch die Perspektiven politischer Theorien und juristischer Doktrinen zu verschränken. Erst mit diesem begrifflichen Schritt zur Weltgesellschaft kann der Individualismus der IG bzw. GG-Theorien überwunden werden (vgl. Wiener 2010). Die mit hoch aggregierten Gesellschaftstheorien notwendig einhergehende Abstraktion muss zwar mit Konkretheitsverlusten erkauft werden, die aber durch neue Respezifizierungsmöglichkeiten kompensiert werden können.

\subsection{Krise und Kritik}

Nach wie vor gibt es - zumindest in Deutschland - zwei holistische Theorieangebote, die das leisten könnten: Die funktionalistische Systemtheorie und die Kritische Theorie. Ich möchte mich hier für die letztere stark machen ${ }^{17}$, weil nur sie eine nichtempiristische Vermittlung von Normativismus und Realismus nicht schon von vornherein ausschließt. ${ }^{18}$ Für eine solche Vermittlungsleistung ist nach wie vor »Das Kapital« von Marx paradigmatisch (siehe auch unter Abschnitt 4, vgl. außerdem Brunkhorst 2011). Zu »bewahren wäre« von Marx vor allem, so Niklas Luhmann treffend, »die Auffassung der Gesellschaft als eines sich selbst abstrahierenden, kategorisierenden, thematisierenden Sozialsystems, also die Negation einer gesellschaftsexternen Geistigkeit, eines transzendentalen Bewusstseins, das sich selbst die Gesellschaft erklärt« (Luhmann 1973: 31). Ganz ähnlich Habermas, der die bleibende Bedeutung des Marxismus mit Marx und Horkheimer ebenfalls darin sieht, dass »die kritische Gesellschaftstheorie« sich dadurch vom transzendentalen Bewusstsein absetzt, dass

»sie sich der Selbstbezüglichkeit ihres Geschäfts inne wird; sie weiß, dass sie dem objektiven Lebenszusammenhang, den sie zu erfassen trachtet, durch die Akte der Erkenntnis hindurch auch zugehört. Der Theorie bleibt ihr Entstehungskontext nicht äußerlich, sie nimmt ihn reflexiv in sich auf [...]. Dasselbe gilt für den Verwendungskontext « (Habermas 1981: 590-591; ähnlich Marcuse 1962: 224, 230).

Die theoretische Schwäche selbstbezüglicher Erkenntnis ist ihre praktische Stärke:

»Eine Gesellschaftstheorie, die für ihre Grundbegriffe Universalität beansprucht, ohne sie einfach konventionell an ihren Gegenstand herantragen zu können, bleibt in der Selbstbe-

17 Für eine Perspektive, die an die Systemtheorie anschließt, aber (wie ich selbst) Querverbindungen nicht ausschließt, siehe Kessler (2010).

18 Luhmann operiert zwar im Unterschied zum naiven Empirismus, zu strategischen Handlungs- und Spieltheorien und dem Paradigma der Trivialmaschine mit einer Reflexionsfigur, die der non-trivialen Mechanik von Subjekten und sozialen Systemen angemessen ist, und er verwendet anstelle des Postmodells der Kommunikation (Übertragung von Sender auf Empfänger, Nachrichtentransport, Austausch) einen Kommunikationsbegriff, der nicht von vornherein die dreidimensionale Komplexität sozialer Kommunikation unterbietet, die (mit Karl Bühler) Mitteilung (Sprechakt-Modus), Information (propositionaler Gehalt) und Verstehen (Ja/Nein-Stellungnahme zu einem Sprechaktangebot) unterscheidet. Zur Kritik des Postmodells siehe schon Luhmann (1964: 191ff.); vgl. auch Luhmann (1984: 191-203) zu Karl-Otto Apel und Habermas und deren Bezug auf Bühler. 
züglichkeit befangen, die Marx am Beispiel des Begriffs der abstrakten Arbeit demonstriert hat « (Habermas 191: 592). ${ }^{19}$

Anders als für Luhmanns technisch neutralisierten soziologischen Beobachter aber ist die reflexive Selbstbezüglichkeit kritischer Theorie nicht nur theoretisch, sondern auch praktisch. Die reflexive Selbstbegründung der Theorie ist nämlich »immer schon « (Heidegger 1977) durch die Perspektive der jeweils betroffenen sozialen Akteure und deren Krisenerfahrung vermittelt. Erst wenn eine Lebenswelt in die Krise gerät, kommt sie als Totalität in den erkennenden Blick (für die Lebenswelt der Wissenschaft: Kuhn 1968). Diesen Blick hat George Lukács (1967) Klassenbewusstsein genannt. ${ }^{20}$ In seiner geschichtlichen Perspektive wird die Gesellschaft als ganze distanzier- und kritisierbar. Nur aus diesem Grund kann eine unvermeidlich selbstbezügliche Theorie der Gesellschaft

»hoffen, der ratio essendi ihrer Gegenstände gewachsen zu sein, wenn Grund für die Annahme besteht, dass der objektive Lebenszusammenhang, in dem sich der Theoretiker selbst vorfindet, dafür sorgt, dass sich ihm die ratio cognoscendi eröffnet« (Habermas 1981: 590).

Kritische Erkenntnis gesellschaftlicher Totalität ist nämlich dann und nur dann möglich, wenn die »realen Abstraktionen « der Gesellschaft »für die Betroffenen selbst ein Problem schaffen und als »objektive Herausforderungen« hervortreten, "angesichts deren die Lebenswelt im ganzen problematisch « (Habermas 1981: 592, 590, Hervorh. dort) wird. Krisen haben eine symptomatische Wahrheit (Žižek 2001: 177-178). ${ }^{21}$ Während die Systemtheorie den normativen Begriff der Krise, in dem die objektive Struktur der Gesellschaft von den Akteuren selbst in ihrer Negativität als soziale Differenz, als Ausbeutung, Unrecht und Unterdrückung erfahren wird, durch den technischen Begriff des sachlichen Risikos substituiert, das komplexen Systemen aus einer unüberschaubaren und unkontrollierbaren Umwelt droht, bleibt die kritische Theorie Krisentheorie und negativ. ${ }^{22}$ Statt sich affirmativ am jeweils bestehenden sense of justice (Rawls) zu orientieren, nutzt sie die verallgemeinernde Kraft der Negation, die im »sense of injustice« (Moore 1978: 47) offenbar wird, den ausgebeutete

19 Mit Bezug auf Karl Marx (1953: 25): »Die einfachste Abstraktion also, welche die moderne Ökonomie an die Spitze stellt und die eine uralte und für alle Gesellschaftsformen gültige Beziehung ausdrückt, erscheint doch nur in dieser Abstraktion praktisch wahr als Kategorie der modernsten Gesellschaft.«

20 Auch der Heideggermarxismus hat den Zusammenhang von Totalitätserkenntnis und Krisenerfahrung scharf und einleuchtend herausgearbeitet, siehe vor allem das Frühwerk von Herbert Marcuse.

21 Die Vermittlung von Krisenerfahrung und Totalitätserkenntnis unterscheidet (neben der negativen Theorie der Gerechtigkeit) die hier vorgeschlagene Konzeptualisierung kritischer Theorie von ihrer Grundlegung in einem überpositiven Recht (Rainer Forst) ebenso wie von der pragmatistisch »praxeologischen « Demokratietheorie (Peter Niesen), mit der sie die These der demokratischen Konstitution allen Rechts und aller Rechte teilt. Vgl. Forst (2010) und Niesen (2010).

22 Siehe nur: Horkheimer (1932), Habermas (1976b). Zum Vorrang der Negation systematisch: Marcuse (1941). Zum jüngsten Stand der Diskussion vgl. auch Celikates (2010). 
Klassen, unterdrückte Völker und exkludierte Populationen selbst artikulieren. ${ }^{23}$ »Negative Gefühle«, sofern es sich um moralische Gefühle wie Entrüstung oder den Biss des Gewissens handelt, zeichnen sich vor den affirmativen durch einen besonderen »kognitiven Gehalt« (Habermas 1999: 277) aus, der in ihrer Intersubjektivität liegt. ${ }^{24}$ Wer sich über (die eigene) Ausbeutung entrüstet, hat einen Grund, der sich auch von jedem beliebigen Anderen teilen (und im Zweifelsfall auf seine Richtigkeit prüfen) lässt. Deshalb ist das moralische Gefühl der Kränkung, ist die Sklavenmoral kein Ressentiment (Wingert 1993: 79). Wenn, so Barrington Moore, ausgebeutete Arbeiter oder unterdrückte Populationen ihre »rejection of suffering and oppression" zum Ausdruck bringen, sagen sie so etwas wie: »Workers of the world, unite! «, »A fair day's pay for a fair day's work! «, »Land to the tillers! « und »Liberty, Equality, Fraternity!« (Moore 1978: 81). Sie nutzen dabei selbst den Universalismus der Negation, ohne den auch die theoretische Erkenntnis der Gesellschaft nicht möglich wäre.

\section{Ein Paradigma}

\subsection{Klassenkampf als Lernprozess}

Marx zeigt im »Kapital« exemplarisch, dass die Probleme sozialer Differenzierung (Ausbeutung, Unrecht, Unterdrückung) nicht etwa, wie Luhmann vermutet, zusammen mit der starr hierarchisierten, alteuropäischen Stratifikationsordnung hinter der versachlichten Fassade von Spezialeinrichtungen verschwinden und »just trivial« werden (Luhmann 1997b: 67, 70). Im Gegenteil, soziale Klassenkonflikte werden mit

23 Zur Verallgemeinerungsleistung der Negation vgl. Kesselring (1984). Auch Lutz Wingert erinnert an den Vorrang der Negation, wenn er rationalen Konsens als Negation der Negation bestimmt: »Entkräftung wechselseitiger Kritik « (Wingert 1993: 215, vgl. auch 221). Zur konstitutiven Rolle von Unrechtserfahrungen für die Entwicklung des Gerechtigkeitsbewusstseins vgl. Piaget (1973: 311). Auch Kant macht sich, sowie er sich Politik und Geschichte zuwendet, immer wieder die reflexiv universalistische Dynamik der Negation zunutze, siehe nur Kant (1977: 216): »dass eine Rechtsverletzung«, nicht aber eine Gerechtigkeit »an einem Platz der Erde an allen gefühlt wird«. Moral universalisiert sich erst, wenn sie erfährt, dass die Kolonialherren »Unrecht wie Wasser trinken«. Die Entdeckung dieses Potentials negativer Gerechtigkeit geht vermutlich bis auf die prophetischen Theodizeen des Leids zurück (Weber 1978: 246). Vgl. auch zur Bedeutung der Substitution der philosophischen »Perfektionsgestalt« des Menschen durch dessen »Defizitgestalt« für die Universalisierung des Gerechtigkeitsbegriffs Cohen (1923: 321).

24 Auch Adorno macht sich diesen kognitiven Gehalt negativer moralischer Gefühle zunutze, freilich um gleich praktische Begründung durch Evidenz zu ersetzen: »Moralische Fragen stellen sich bündig [...] in Sätzen wie: Es soll nicht gefoltert werden; es sollen keine Konzentrationslager sein [...]. Wahr sind die Sätze als Impuls, wenn gemeldet wird, irgendwo sei gefoltert worden. Sie dürfen sich nicht rationalisieren; als abstraktes Prinzip gerieten sie sogleich in die schlechte Unendlichkeit ihrer Ableitung und Gültigkeit. [...] Der Impuls, die nackte physische Angst und das Gefühl der Solidarität mit den, nach Brechts Worten, quälbaren Körpern, der dem moralischen Verhalten immanent ist, würde durchs Bestreben rücksichtsloser Rationalisierung verleugnet; das Dringlichste würde abermals kontemplativ, Spott auf die eigene Dringlichkeit« (Adorno 1966: 279). 
der Emergenz des modernen, ganz und gar vorbildlosen Kapitalismus wie nie zuvor dynamisiert. Sie lassen alles »Ständische und Stehende« (Marx/Engels 1848) verdampfen und verlieren ihren eingeborenen Charakter. Jetzt erst treten sie in den Krisen der modernen Gesellschaft aus dem abgeschatteten Hintergrund eherner Verhältnisse hervor und lassen die Gesellschaft als »einen umwandlungsfähigen und beständig im Prozess der Umwandlung begriffenen Organismus « (Marx 1867: 16) erscheinen. Die neuen Klassengegensätze entstehen nämlich nur noch infolge der Umstellung der Gesellschaft von sozialer Stratifizierung auffunktionale Differenzierung und müssen deshalb im Vollzug funktionaler Differenzierung und damit in Abhängigkeit vom rasanten Komplexitätswachstum der modernen Gesellschaft tagtäglich neu produziert und individuell zugerechnet werden. Während alle Wellen gesellschaftlicher Veränderung, die von den Stürmen der großen religiösen Revolutionen und Reformationen des 12., 16. und 17. Jahrhunderts in Bewegung gesetzt worden sind, sich an der fortbestehenden Stabilität der Schichtungsordnung wieder und wieder gebrochen haben, haben die Verfassungsrevolutionen des 18. Jahrhunderts mit der ständisch erstarrten Klassenstruktur die Differenz von Stabilität und Veränderung »kollabieren« lassen (vgl. Luhmann 1997a: 498-499) und die Klassenverhältnisse in eine unaufhörliche Bewegung versetzt: Die ökonomische Bewegung am Arbeitsmarkt lässt Einstellungsund Entlassungswelle auf Einstellungs- und Entlassungswelle folgen, und beide werden beständig von immer wieder neu anrollenden Migrationswellen überlagert; die sozialen und politischen Bewegungen organisieren sich im Klassenkampf um Subsistenz und Profit, Macht und Hegemonie, Recht und Verfassung, Ideen und Interessen. Im Verlauf dieser Kämpfe, die sich in rasch wechselnden Konstellationen vollziehen, setzt die moderne Gesellschaft bis dahin ungeahnte Lernchancen frei, so dass sich die gesellschaftlichen Folgen des Klassenkampfes schließlich den Akteuren selbst (von oben und von unten) als »fortwährende Revolutionierung [...] sämtlicher gesellschaftlichen Verhältnisse« (Marx/Engels 1848) darstellen. ${ }^{25}$ Die Entwicklung von kollektiven Lernprozessen folgt einer spezifischen Krisendynamik: Der Klassenkonflikt von Kapital und Arbeit, der sich infolge der Ausdifferenzierung des selbstreferentiell geschlossenen, deshalb umweltblinden Wirtschaftssystems herausbildet, überfordert das auf Freiheit und Gleichheit gemünzte Legitimationspotential des bürgerlich-parlamentarischen Verfassungsstaats strukturell, so dass es infolge von ökonomischen Systemkrisen vollends aufgezehrt wird. Die objektive Systemkri$s e$, die von den vielen zyklischen Wirtschaftskrisen über die wenigen großen Weltwirtschafts- und Finanzkrisen (wie zuletzt 2008-2010) bis zur strukturellen fiscal crisis des Staates (O'Connor 1973) reicht, schlägt dann in eine nur noch normativ auflösbare Legitimationskrise um, in der Klassenkämpfe, Konflikte und Krisenerfahrungen sich mit Totalitätserkenntnis und normativen Lernprozessen verschränken. An diesen Mechanismus schließt die leninistische Revolutionstheorie ebenso an wie der sozialdemokratische Reformismus. Die Transformation des elitären bürgerlichen Parlamentarismus des 19. Jahrhunderts in die soziale Massendemokratie und den bü-

25 Zum inneren Zusammenhang von Klassenkampf und normativem Lernprozess Eder (1983: $25 ; 1987: 104$.$) .$ 
rokratischen Sozialismus des 20. Jahrhunderts - ein exemplarischer normativer Lernprozess $^{26}$ - kann mit Hilfe dieser Annahmen zwar nicht hinlänglich, aber doch im Ansatz erklärt werden.

\subsection{Abstraktion und Respezifizierung}

Die eigentlich politische Transformation vom liberalen zum sozialen Interventionsstaat (auch der liberale Klassenstaat war Interventionsstaat), die Globalisierung staatlicher Organisation und die Verrechtlichung und Konstitutionalisierung der Weltgesellschaft aber können mit dem Verweis auf kapitalorientierte Konflikte (nicht nur zwischen Kapital und Arbeit, auch zwischen Kapital und Kapital, Stadt und Land usw.) so wenig erklärt werden wie die Schubkraft, Organisation und Transformation sozialer Bewegungen (Arbeiterbewegung, Frauenbewegung, Friedensbewegung, religiöser Fundamentalismus usw.). An diesen Fällen wird evident, dass das marxistische Paradigma nur ein Paradigma ist, das nicht eindimensional verallgemeinert werden darf, da es nur kapitalorientierte Konflikte, aber weder staatsorientierte Konflikte noch glaubensorientierte Konflikte hinlänglich erklären kann. ${ }^{27}$

Während kapitalorientierte Konflikte, die sich zum Gegensatz der materiellen und ideellen Interessen antagonistischer Großgruppen zuspitzen können, in Folge der Ausdifferenzierung und erweiterten Reproduktion des kapitalistischen Wirtschaftssystems entstehen, entstehen staatsorientierte Konflikte infolge der Ausdifferenzierung und Globalisierung staatlich organisierter Zwangs- und Administrativmacht (seit dem 16. Jh.) Solche Konflikte werden nicht nur zwischen Machthabern und Machtunterworfenen (Tilly 1995), zwischen Volk und Machtblock (Laclau 1995), zwischen Politik und Polizei (Rancière 2002) ausgetragen, sondern auch zwischen Staaten oder zwischen Staaten und global operierenden Organisationen der Mafia, des Kapitals, der Arbeit oder des religiösen Fundamentalismus.

$\mathrm{Zu}$ einem dritten Typus spezifisch glaubensorientierter Konflikte kommt es - so lassen es zumindest die jüngeren Forschungen zur Rechtsgeschichte der päpstlichen und protestantischen Reformationen (Revolutionen) vermuten ${ }^{28}$ - erstmals infolge der frühen Ausdifferenzierung des Rechtssystems, dessen individualisierende Folgen sich seit der frühen Neuzeit nicht mehr in das System klerikaler Zwangsorganisatio-

26 Es gibt auch »Lernprozesse mit tödlichem Ausgang« (Kluge 1999). Lernen und Verlernen gehören zusammen. Nicht nur sind jederzeit regressive oder negative Lernprozesse möglich, jeder Lernprozess ist zugleich ein Verlernprozess. Wer hypothetisch zu denken gelernt hat, musste das magische Wissen seiner Kindertage zunächst einmal verdrängen und vergessen. Aber so oder so gilt von Menschen dasselbe wie von Gesellschaften: »Gesellschaften können nicht nicht lernen« (Jürgen Habermas, mündliche Äußerungen, zitiert nach Eder (2009: 67)).

27 Zur Unterscheidung von kapital- und staatsorientierten Konflikten: Tilly (1995: 36-39). Vgl. auch ten Brink (2008), der entsprechend zwischen Geopolitik und Kapitalkonkurrenz unterscheidet. Zur erweiterten Typologie struktureller Klassenkonflikte Brunkhorst (2011).

28 Vgl. Witte (2002); Brundage (1994; 1995); Fried (1974); Berman (1983a; 1983b); Blickle (2003). 
nen integrieren ließen. Das dabei freigesetzte Potential voluntaristisch-korporativer Selbstorganisation, das zuerst von den protestantischen Sekten, Kirchen und Kapitalorganisationen (z. B. zur Bildung von neuartigen private-public partnerships wie den schon erwähnten East India Companies) genutzt wurde, stand danach für die Bildung beliebiger sozialer Bewegungen zur Verfügung. Diese stellen ein gutes Beispiel für einen in revolutionären Klassenkämpfen und Glaubenskriegen initiierten, erfolgreichen Lernprozess mit immensen und kaum zu überschätzenden Folgen für die Organisation der Freiheit dar. ${ }^{29}$

Im Zuge der Globalisierung scheint es infolge der Überforderung des systemischen Integrationspotentials bei der funktionalen Differenzierung von Recht, Wirtschaft und Politik zu einer vierten, strukturellen Konfliktkonstellation zu kommen, deren Frontlinie zwischen eingeschlossen und ausgeschlossenen Populationen (Neves 1992), zwischen den Vernetzten und den Unvernetzten (Castells 2001) verläuft. Die Globalisierung der letzten fünfzig Jahre hat gleichzeitig den bis dahin auf den Nationalstaat beschränkten Anspruch auf Ausschluss von Ungleichheit (Stichweh 2000: 52) universalisiert und alle Funktionssysteme und kulturellen Wertsphären der Gesellschaft in Weltsysteme verwandelt. Auch die noch rein national institutionalisierten Systeme wie das Erziehungssystem haben überall dieselben strukturellen Merkmale, dieselbe Stufenfolge der Schulabschlüsse und -übergänge, und sie verändern sich alle zur selben Zeit und in dieselbe Richtung, die z. B. durch empirische Untersuchungen wie die Pisa-Studie und andere UNESCO-Studien vorgegeben werden (Meyer 1997). Als Weltrecht, Weltpolitik und Weltwirtschaft sind die Funktionssysteme funktional auf universale Inklusion (offener Zugang für jedermann) angewiesen. Gleichzeitig aber sind sie mit dieser Aufgabe offensichtlich überfordert und erzeugen wachsende Überschusspopulationen (Arendt 1976). Die teils initiativen, teils nachwachsenden staatsartigen internationalen Organisationen und die kooperierende Staatengemeinschaft sind bislang nicht in der Lage, hier für Ausgleich zu sorgen. Die Entwicklung scheint auf eine absehbare Verschärfung der Klassenkonflikte zwischen über- und unterintegrierten Populationen hinauszulaufen, die sich heute schon in den Banlieues der reichen Welt abzeichnet und sich überall mit sozialen Klassenkämpfen, nationalen Befreiungsbewegungen und religiösem Fundamentalismus verschränkt und vermengt (integrationsorientierte Konflikte) (Neves 1999).

29 Siehe nur die vielzitierten (und sogar viel gelesenen) Beobachtungen von Alexis de Tocqueville (1990). 
Die folgende Tabelle fasst das Ergebnis der abstrahierenden Respezifizierung des marxistischen Paradigmas zusammen:

Tabelle 1: Sozialer Konflikt (»Klassenkampf«) und Gesellschaftsstruktur

\begin{tabular}{|l|l|l|}
\hline Funktionale Differenzierung & Lebensweltliche Wirkung & Sozialer Konflikt \\
\hline $\begin{array}{l}\text { Rechtssystem } \\
\text { (seit 12. Jahrhundert) }\end{array}$ & Individualisierung & $\begin{array}{l}\text { Glaubensorientierte Kon- } \\
\text { flikte }\end{array}$ \\
\hline $\begin{array}{l}\text { Politisches System } \\
\text { (seit 16. Jahrhundert) }\end{array}$ & $\begin{array}{l}\text { Transformation »lebendiger } \\
\text { Volksmacht« (Arendt) in to- } \\
\text { te Zwangs- und Administra- } \\
\text { tivmacht }\end{array}$ & Staatsorientierte Konflikte \\
\hline $\begin{array}{l}\text { Ökonomisches System } \\
\text { (seit 18. Jahrhundert) }\end{array}$ & $\begin{array}{l}\text { Transformation »lebendi- } \\
\text { ger« in »tote Arbeit« (Marx) }\end{array}$ & Kapitalorientierte Konflikte \\
\hline $\begin{array}{l}\text { Globalisierung von Recht, } \\
\text { Politik und Wirtschaft } \\
\text { (seit 20. Jahrhundert) }\end{array}$ & $\begin{array}{l}\text { Exklusion von Überschus- } \\
\text { spopulationen }\end{array}$ & $\begin{array}{l}\text { Integrationsorientierte Kon- } \\
\text { flikte }\end{array}$ \\
\hline
\end{tabular}

\subsection{Probleme}

Die moderne Gesellschaft lernt mehr als jede frühere Gesellschaftsformation durch Klassenkämpfe und soziale Konflikte. Dabei widerstreiten, überschneiden und verbinden sich kapitalorientierte mit staatsorientierten Konflikten, Glaubenskriegen und Inklusionskämpfen auf vielfältige Weise. Die Entwicklung des Nationalstaats, oder besser mit Tilly, des nationalen Staats ${ }^{30}$ zum demokratischen Verfassungsstaat hat (zumindest in den glücklicheren, reicheren und mächtigeren Segmenten der Staatenwelt) Klassenkämpfe und soziale Konflikte in Kämpfe ums Recht verwandelt, die im Recht (mit legalen oder illegalen Mitteln) ausgetragen werden. Die reflexive Konstitutionalisierung des Klassenkampfes hat zu einer Verstetigung und Expansion normativer Lernprozesse geführt - greifbar z.B. in der prospektiven Beschreibung des Parlamentarismus als permanente legale Revolution um 1848 (Maus 1990).

Sie legt es, gerade mit Blick auf globale Verrechtlichungs- und Vergesellschaftungsprozesse, nahe, nicht nur statisch zwischen Typen ${ }^{31}$, sondern auch zwischen

30 Den nationalen Staat definiert Tilly durch zentralisierte Territorialherrschaft über und (aktiv oder passiv) durch eine eigene Population von Staatsangehörigen. Der nationale Staat (national state) ist von dem mittlerweile kaum noch (außer vielleicht in Dänemark) existierenden Nationalstaat (nation state) mit einer »strong linguistic, religious and symbolic identity « (Tilly 1990: 3) scharf zu unterscheiden.

31 Vgl. Möllers (2003); ähnlich auch Maus (1994); Arendt (1986: 187-191); jüngst vgl. Haller et al. (2010). Siehe neben der »Einführung« von Haller dort insbesondere die Unterscheidung jeweils von »rechtlichem « und »demokratischem « Konstitutionalismus, Rechte »von unten« vs. Rechte »von oben« und »Demokratie durch Recht« vs. »Recht durch Demokratie« in den Beiträgen von Ballamy, Dellavalle und Brunkhorst in Haller et al. (2010). 
Entwicklungsphasen revolutionärer und evolutionärer Konstitutionalisierung zu unterscheiden. Revolutionäre Konstitutionalisierung bringt in extrem kurzen Zeiträumen - der punctualization langer Perioden des equilibrium zwischen Organismen und Umwelt in der organischen Evolution vergleichbar ${ }^{32}$ - einem neuen institutionellen Rahmen der Gesellschaft hervor. Ein solcher institutioneller Rahmen stellt die Verfassung der Gesellschaft im umfassenden, formellen und materiellen, wissenschaftlichen und alltäglichen Sinn dar. Sie begrenzt die fortlaufende soziale Evolution und damit die Beliebigkeit der selektiven Adaption der Gesellschaft an wechselnde Umwelten normativ und schließt bestimmte Anpassungsleistungen (z. B. solche autoritärer Regimes, die elementare Menschenrechtsverletzungen, Verstöße gegen die egalitäre Menschenwürde usw. einschließen) auch dann aus, wenn sie den größeren evolutionären Erfolg versprechen - was wiederum der Rolle der Arten spezifizierenden Baupläne, die in der organischen Evolution als internal constraints adaptiver Beliebigkeit fungieren, analog ist. ${ }^{33}$ Aktuelle Beispiele sind etwa die Debatten zum Luftsicherungsgesetz oder zur »rechtsstaatlichen« Folter.

Revolutionen institutionalisieren kollektive Lernprozesse, die in Verfassungs- und Rechtstexten zu objektivem Geist gerinnen. Revolutionäre Umbrüche folgen dabei in der Regel einer Entwicklungslogik, die zumindest bestimmte notwendige Bedingungen der Entwicklung von A zu B spezifiziert, aber niemals, dass B auf A folgen muss und nicht genauso gut $\mathrm{C}, \mathrm{D}, \ldots \mathrm{N}$, die $\mathrm{B}$ ausschließen, folgen könnten. ${ }^{34}$ So setzt z.B. funktionale Differenzierung Stratifizierungs- und Segmentierungsleistungen voraus, die schon vorhanden sein müssen, wenn es zu funktionaler Differenzierung kommen soll. Die Einführung universeller und gleicher Rechte andererseits setzt eine evolutionär ausdifferenzierte Rechtsform voraus, in der Rechte überhaupt spezifiziert und normiert werden können. ${ }^{35}$

Revolutionären Umbrüchen folgen in der Regel ruhigere Entwicklungsphasen evolutionärer Konstitutionalisierung, die eher dem klassisch Darwinistischen Muster langsamen adaptiven Wandels durch kommunikative Zufallsvariation und strukturelle Selektion entsprechen. ${ }^{36}$ So hinterließ die Französische Revolution, obwohl ihre Niederlage 1815 totaler nicht hätte sein können, einen Bauplan normativer constraints, der eine Fortentwicklung der Staatenwelt in nicht-konstitutionellen Bahnen unmöglich erscheinen ließ, wie Volker Sellin (2001) am Beispiel der fälschlich so

32 Mayr (1992); Gould/Lewontin (1979); Eldredge/Gould (1972); Gould (1978; 2002).

33 Vgl. Gould (1984; 2002: 290, 753, 884-895). Zur Rolle normativer constraints in der sozialen Evolution finden sich erste Überlegungen in Brunkhorst (2010b).

34 Vorausgesetzt ist auch nicht, dass es nur eine Evolution gibt. Natürlich gibt es immer eine unbegrenzte Vielzahl möglicher Co-Evolutionen, und es ist eine empirische Frage, welche tatsächlich evoluieren.

35 Vgl. auch Habermas (1992: 167-187), der hier ein wenig beachtetes, aber für die ganze Konstruktion der Diskurstheorie des Rechts tragendes Argument ausführt.

36 Weil die Systemtheorie von der Rolle revolutionärer Lernprozesse (und damit auch von Klassenkämpfen) in der sozialen Evolution abstrahiert, muss sie die gründende Leistung von Verfassungen auf ein reines Überbauphänomen, das falsche Selbstverständnis der Machbarkeit von Geschichte reduzieren (Luhmann 1990). Dasselbe Problem einer schlechten Abstraktion (Hegel) stellt sich, wenn man die Evolution auf die »gegenseitige Konstitution von Semantik und Sozialstruktur« reduziert (vgl. Kessler 2010). 
genannten Restauration gezeigt hat. ${ }^{37}$ Das alles lässt sich auch gut auf aktuelle Debatten im Völkerrecht und in den IB/der IPT übertragen. So wird im Internationalen Recht beobachtete Konstitutionalisierung des Völkerrechts zumeist als evolutionäre Konstitutionalisierung verstanden (Frowein 2001; Tomuschat 1995). Dem entspricht in den IB/der IPT die Theorie der invisible constitution (Wiener 2008) bzw. des hidden constitutional discourse (Tully 2002). Während jedoch einige Völkerrechtler auch revolutionäre Konstitutionalisierungsleistungen in der Weltgesellschaft des 20. Jahrhunderts beobachten, die sie der UN-Charta (1945), dem Vertrag von Amsterdam (1997) oder dem massiven und plötzlichen Konstitutionalisierungsschub von 1989 (Fassbender 2009; Bogdandy 2009; Peters 2006) zurechnen, fehlen vergleichbare Studien in den IB/der IPT. Es läge jedoch gerade für eine kritische Theorie internationaler Beziehungen nahe, den Rawlsschen overlapping consensus und das Überlegungsgleichgewicht dem evolutionären Konstitutionalismus zuzuordnen, während die normativen Ansprüche der Betroffenen auf Erlösung vom gesellschaftlichen Übel, die in den Rechteerklärungen von 1789 und 1948 zu Geschichtszeichen geworden sind, den normativen constraints des revolutionären Konstitutionalismus und den progressiven Einsichtsgewinnen kollektiver Lernprozesse zuzuordnen wären (passend zur Unterscheidung, die Forst 2010 unternimmt).

Die Dialektik von Revolution und Evolution ist in demokratischen Verfassungsregimes keineswegs zur Ruhe und zum kristallinen Stillstand gekommen. Verfassungen bringen, solange sie noch einen Funken normativer Kraft haben, soziale Konflikte und Klassenkämpfe als Medien kollektiven Lernens nicht zum Verschwinden, sondern ermöglichen im Gegenteil die organisierte Fortsetzung des Kampfes in »rechtlich institutionalisierten rechtsfreien Räumen « (Maus 1990, 371). Diese nationalstaatliche und bloß partikulare Lösung aber scheint in einer Welt, die nicht nur internationale Gemeinschaft, sondern auch funktional differenzierte Weltgesellschaft ist, nicht mehr verfügbar oder wie Wolfgang Streeck (2010: 172) jüngst im »Leviathan « schrieb, es spreche fast alles dafür, »dass am Beginn des 21. Jahrhunderts die Daueraufgabe der Stabilisierung der kapitalistischen Marktwirtschaft die Kräfte des Nationalstaats endgültig erschöpft« habe. Auf der Ebene der Internationalen Gemeinschaft aber gibt es - trotz aller Verrechtlichung, Konstitutionalisierung und Weltstaatlichkeit $^{38}-$ zu dieser Leistung des nationalen Staats bislang kein funktionales Äquivalent. Es wird dringend benötigt, aber ob die Globalisierung des Klassenkampfes, die wir derzeit vor allem von oben beobachten können (Emergenz einer transnational herrschenden Klasse, und auf der andern Seite: nur multitude, kein Volk, keine Klasse, keine Kirche, allenfalls fundamentalistische Sekten ${ }^{39}$ ), einen weiteren Lernprozess in Gang setzt, der internationale Institutionen hervorbringen könnte, die de-

37 Mit Hilfe solcher Studien lässt sich etwa Kants berühmter Satz, die Revolution sei ein Ereignis gewesen, das sich nicht vergesse, von seiner heute unplausibel gewordenen, vermögenspsychologischen Begründung ablösen und im Rahmen einer empirischen Evolutionstheorie neu begründen.

38 Insbesondere zu letzterer Albert/Stichweh (2007); Shaw (2000); Chimni (2004).

39 Vgl. Brunkhorst (2002: 153); zur Überformung nationaler durch inter- und transnationale Herrschaft siehe auch Deitelhoff (2010). 
mokratisch und mächtig genug sind, um das doppelte Problem der Stabilisierung der Weltwirtschaft und der Konstitutionalisierung globaler Klassenkämpfe zu lösen, ist eine offene Frage.

\section{Zusammenfassung}

Ich war von einer sehr schematischen Konfrontation normativistischer und realistischer Paradigmen in der IPT ausgegangen. Es zeigte sich eine komplementäre Schwäche (unterkomplexer Realismus vs. realitätsferner Normativismus), die sich aber durch Rückgriff auf die Bestände holistischer Gesellschaftstheorien überwinden lässt. Das wurde am Beispiel eines Beitrags von Parsons zur IPT zunächst exemplarisch erläutert. Danach habe ich dann den Versuch eines Neustarts kritischer Gesellschaftstheorie auf alter Festplatte skizziert. Zu diesem Zweck wurde die Integration von Klassenkonflikt (als dem Medium normativer Lernprozesse) und Evolution (als dem Medium funktionaler Anpassungsprozesse) am Beispiel von Marx »Das Kapital« kurz entwickelt. Das Ergebnis wurde dann zu einem Schema abstrahiert, das es erlauben soll, die komplexe Klassen- und Konfliktkonstellation der Weltgesellschaft $\mathrm{zu}$ rekonstruieren, empirisch zu erfassen und in ihren strukturellen (systemischen) Bezügen zu den Krisentendenzen der modernen kapitalistischen Gesellschaft erkennbar zu machen. Daraus ergeben sich dann erste Thesen zu weltgesellschaftlichen Krisenszenarien, Strukturproblemen und Klassenkonstellationen. Eine entsprechende Theorie mitsamt der zugehörigen Zeitdiagnose muss freilich erst noch in größerem Format ausgearbeitet werden.

\section{Literatur}

Adorno, Theodor W. 1966: Negative Dialektik, Frankfurt a. M.

Albert, Mathias/Stichweh, Rudolf(Hrsg.) 2007: Weltstaat und Weltstaatlichkeit, Wiesbaden. Arendt, Hannah 1986: Über die Revolution, München.

Arendt, Hannah 1976: Origins of Totalitarianism, San Diego, CA.

Bargu, Banu 2010: The Problem of the Republic in Marx and Machiavelli, New York, unv. Ms. Barnett, Michael/Sikkink, Kathryn 2008: From International Relations to International Global Society, in: Reus-Smit, Christian/Snidal, Duncan: Oxford Handbook of International Relations, Oxford, 62-81.

Beck, Ulrich o. J.: National, International, Transnational, Kosmopolitisch - Perspektiven der Ungleichheitsforschung, in: http://www.soziale-ungleichheit.de/tagungen/sektion/transnationalisierung/praes/Beck_Transnationalisierung_Praes.pdf; 10.10.2010.

Benjamin, Walter 1974: Über den Begriff der Geschichte, in: Benjamin, Walter: Illuminationen. Ausgewählte Schriften, Band 1, Frankfurt a. M.

Berman, Harold 1983a: Law and Revolution: The Formation of the Western Legal Tradition, Cambridge, MA.

Berman, Harold 1983b: Law and Revolution II: The Impact of the Protestant Reformations on the Western Legal Tradition, Cambridge, MA.

Blickle, Peter 2003: Von der Leibeigenschaft zu den Menschenrechten. Eine Geschichte der Freiheit in Deutschland, München. 
Brandt, Rainhard 1982: Das Titelblatt des Leviathan und Goyas El Gigante, in: Bermbach, Udo/ Kodalle, Klaus-Michael (Hrsg.): Furcht und Freiheit, Opladen, 203-231.

Bredekamp, Horst 1999: Thomas Hobbes Visuelle Strategien, Berlin.

Brundage, James A. 1994: The Rise of the Professional Jurist in the Thirteenth Century, in: Syracuse Journal of International Law and Commerce 20, 185-190.

Brundage, James A. 1995: Medieval Canon Law, London.

Brunkhorst, Hauke 2002: Solidarität. Von der Bürgerfreundschaft zur globalen Rechtsgenossenschaft, Frankfurt a. M.

Brunkhorst, Hauke 2008: Kritik am Dualismus des internationalen Recht-Hans Kelsen und die Völkerrechtsrevolution des 20. Jahrhunderts, in: Kreide, Regina/Niederberger, Andreas (Hrsg.): Transnationale Verrechtlichung. Nationale Demokratien im Zeitalter globaler Politik, Frankfurt a. M., 30-63.

Brunkhorst, Hauke 2009: There Will Be Blood. Konstitutionalisierung ohne Demokratie?, in: Brunkhorst, Hauke (Hrsg.): Demokratie in der Weltgesellschaft (Sonderheft Soziale Welt), 111-113.

Brunkhorst, Hauke 2010a: Cosmopolitanism and Democratic Freedom, in: Thornhill, Chris/ Ashenden, Samantha (Hrsg.): Legality and Legitimacy - Normative and Sociological Approaches, Baden-Baden, 171-196.

Brunkhorst, Hauke 2010b: Legitimationsverhältnisse - Eine Replik auf Ingeborg Maus, in: Eberl, Oliver/Niesen, Peter (Hrsg.): ohne Titel, i.E.

Brunkhorst, Hauke 2011: The Return of Crisis, in: Kjaer, Poul/Teubner, Gunther (Hrsg.): The Financial Crisis in Constitutional Perspective: The Dark Side of Functional Differentiation, Oxford, i. E.

Brunkhorst, Hauke/Voigt, Rüdiger (Hrsg.) 2008: Rechts-Staat. Hans Kelsens Rechts- und Staatsverständnis, Baden-Baden.

Castells, Manuel 2001: Das Informationszeitalter, Band 3, Wiesbaden.

Celikates, Robin 2010: Kritik als soziale Praxis. Gesellschaftliche Selbstverständigung und kritische Theorie, Frankfurt a. M.

Chimni, Bhupinder S. 2004: International Institutions Today: an Imperial Global State in the Making, in: European Journal of International Law 15: 1, 1-37.

Cohen, Hermann 1923: Das soziale Ideal bei Platon und den Propheten, in: Cohen, Hermann: Jüdische Schriften, Band 1, Breslau, 321.

de Tocqueville, Alexis 1990: Über die Demokratie in Amerika, Stuttgart.

Deitelhoff, Nicole 2010: Parallele Universen oder Verschmelzung der Horizonte?, in: Zeitschrift für Internationale Beziehungen 17:2, 279-292.

Eberl, Oliver 2007: Demokratie und Frieden. Kants Friedensschrift in den Kontroversen über die Gestaltung globaler Ordnung (Universität Bremen: Dissertation), Bremen.

Eder, Klaus 1983: Collective Learning Processes and Social Evolution: Towards a Theory of Class Conflict in Modern Society, in: Tidskrift för Rätssociologi 1, 23-36.

Eder, Klaus 1987: Learning and the Evolution of Social Systems - An Epigenetic Perspective, in: Schmid, Michael/Wuketits, Franz M. (Hrsg.): Evolutionary Theory in Social Science, Dordrecht, 101-125.

Eder, Klaus 2009: Evolutionstheorie, in: Brunkhorst, Hauke/Kreide, Regina/Lafont, Christina (Hrsg.): Habermas-Handbuch. Leben - Werk - Wirkung, Stuttgart, 65-68.

Eldredge, Niles/Gould, Stephen Jay 1972: Punctuated Equilibria: An Alternative to Phyletic Gradualism, in: Schopf, Thomas J. M. (Hrsg.): Models in Paleobiology, San Francisco, CA, 82-115.

Fassbender, Bardo 2009: The United Nations Charter as the Constitution of the International Community, Leiden, Boston, MA.

Forst, Rainer 2010: Was ist und was soll Internationale Politische Theorie?, in: Zeitschrift für Internationale Beziehungen 17:2, 355-363.

Fried, Johannes 1974: Die Entstehung des Juristenstands im 12. Jahrhundert, Köln. 
Frowein, Jochen A. 2001: Konstitutionalisierung des Völkerrechts, in: BDGVR, Völkerrecht und Internationales Recht in einem sich globalisierenden internationalen System, Heidelberg, 427-447.

Gangl, Manfred (Hrsg.) 2001: Linke Juristen in der Weimarer Republik, Frankfurt a. M.

Gangl, Manfred (Hrsg.) 2008: Das Politische. Zur Entstehung der Politikwissenschaft während der Weimarer Republik, Frankfurt a. M.

Gosepath, Stephan/Merle, Jean-Christoph (Hrsg.) 2002: Weltrepublik, München.

Gould, Stephen Jay 1978: Episodic Change Versus Gradualist Dogma, in: Science and Nature 2, 5-12.

Gould, Stephen Jay 1984: Morphological Challenging by Structural Constraint, in: Palaeobiology 10: 2, 172-194.

Gould, Stephen Jay 2002: The Structure of Evolutionary Theory, Cambridge, MA.

Gould, Stephen Jay/Lewontin, Richard C. 1979: The Spandrels of San Marco and the Panglossian Paradigm: A Critique of the Adaptationist Programme, in: http:/www.aaas.org/spp/dser/ 03_Areas/evolution/perspectives/Gould_Lewontin_1979.shtml; 21.9.2010.

Griffiths, Martin/Roach, Steven C./Solomon, M. Scott 2009: Fifty Key Thinkers in International Relations, 2. Auflage, London.

Gusy, Christoph (Hrsg.) 2000: Demokratisches Denken in der Weimarer Republik, Baden-Baden.

Habermas, Jürgen 1976a: Zur Rekonstruktion des Historischen Materialismus, Frankfurt a. M.

Habermas, Jürgen 1976b: Was heißt heute Krise?, in: Habermas, Jürgen: Zur Rekonstruktion des Historischen Materialismus, Frankfurt a. M., 304-328.

Habermas, Jürgen 1981: Theorie des kommunikativen Handelns, Band 2, Frankfurt a. M.

Habermas, Jürgen 1992: Faktizität und Geltung, Frankfurt a. M.

Habermas, Jürgen 1999: Wahrheit und Rechtfertigung, Frankfurt a. M.

Habermas, Jürgen 2004: Hat die Konstitutionalisierung des Völkerrechts noch eine Chance?, in: Habermas, Jürgen: Der gespaltene Westen, Frankfurt a. M., 113-193.

Haller, Gret/Günther, Klaus/Neumann, Ulfrid (Hrsg.) 2010: Menschenrechte und Volkssouveränität in Europa. Gerichte als Vormund der Demokratie?, Frankfurt a. M., i.E.

Heidegger, Martin 1977: Sein und Zeit, Tübingen.

Held, David 1995: Democracy and the Global Order, Oxford.

Heller, Hermann 1971: Die Souveränität, in: Gesammelte Schriften, Band 2, Leiden, 34.

Herborth, Benjamin/Niesen, Peter (Hrsg.) 2007: Anarchie der kommunikativen Freiheit, Frankfurt a. M.

Herz, John H. 1951: Political Realism and Political Idealism: A Study in Theories and Realities, Chicago, IL.

Höffe, Otfried 1999: Demokratie im Zeitalter der Globalisierung, München.

Hoffmann, Hasso 1997: Bilder des Friedens oder Die vergessene Gerechtigkeit, München.

Horkheimer, Max 1932: Bemerkungen über Wissenschaft und Krise, in: Zeitschrift für Sozialforschung 1-2, 1-7.

Kant, Immanuel 1977: Zum Ewigen Frieden. Ein philosophischer Entwurf, in: Kant, Immanuel: Werke XI, Frankfurt a. M., 195-254.

Kelsen, Hans 1960: Das Problem der Souveränität und die Theorie des Völkerrechts, Nachdruck der 2. Auflage von 1928, Aalen.

Kesselring, Thomas 1984: Die Produktivität der Antinomie. Hegels Dialektik im Lichte der genetischen Erkenntnistheorie und der formalen Logik, Frankfurt a. M.

Kessler, Oliver 2010: Internationale Politische Theorie: Fehlentwicklung oder fehlende Entwicklung der IB?, in: Zeitschrift für Internationale Beziehungen 17:2, 317-334.

Kleingeld, Pauline 2006: Cosmopolitanism, in: Stanford Encyclopedia of Philosophy, http:// plato.stanford.edu/entries/cosmopolitanism/; 21.9.2010.

Kluge, Alexander 1999: Lernprozesse mit tödlichem Ausgang, Frankfurt a.M. 
Koskenniemi, Martti 2002: The Gentle Civilizer of Nations: The Rise and Fall of International Law 1870-1960, Cambridge.

Kreide, Regina 2008: Globale Politik und Menschenrechte. Macht und Ohnmacht eines politischen Instruments, Frankfurt a. M.

Kuhn, Thomas, 1968: Die Struktur wissenschaftlicher Revolutionen, Frankfurt a. M.

Laclau, Ernesto, 1995: Politik und Ideologie im Marxismus. Kapitalismus, Faschismus, Populismus, Berlin.

Lakatos, Imre 1974: The Methodology of Scientific Research Programmes, Philosophical Papers, Band I, London.

Luhmann, Niklas 1964: Funktion und Folgen formaler Organisation, Berlin.

Luhmann, Niklas 1965: Grundrechte als Institution, Berlin.

Luhmann, Niklas 1973: Selbst-Thematisierung des Gesellschaftssystems. Über die Kategorie der Reflexion aus der Sicht der Systemtheorie, in: Zeitschrift für Soziologie 2: 1, 21-46.

Luhmann, Niklas 1984: Soziale Systeme, Frankfurt a. M.

Luhmann, Niklas 1990: Verfassung als evolutionäre Errungenschaft, in: Rechtshistorisches Journal 9, 176-220.

Luhmann, Niklas 1997a: Die Gesellschaft der Gesellschaft, Frankfurt a. M.

Luhmann, Niklas 1997b: Globalization or World Society: How to Conceive of Modern Society?, in: International Review of Sociology 7: 1, 67-79.

Lukács, George 1967: Geschichte und Klassenbewusstsein. Studien über marxistische Dialektik, unautorisierter Nachdruck der 1. Ausgabe von 1923 im Verlag Thomas de Munter, Amsterdam.

Marcuse, Herbert 1941: Reason and Revolution: Hegel and the Origin of the Theory of Society, New York, NY.

Marcuse, Herbert 1962: Vernunft und Revolution, Neuwied.

Marx, Karl 1867: Das Kapital, Band 1, in: http://www.mlwerke.de/me/me23/me23_000.htm, 21.9.2010.

Marx, Karl 1953: Grundrisse der Kritik der politischen Ökonomie, Berlin.

Marx, Karl/Engels, Friedrich 1848: Manifest der Kommunistischen Partei, in: http:// www.mlwerke.de/me/me04/me04_459.htm\#Kap_I; 21.9.2010.

Maus, Ingeborg 1990: Zur Theorie der Institutionalisierung bei Kant, in: Göhler, Gerhard/Lenk, Kurt/Münkler, Herfried/Walther, Manfred (Hrsg.): Politische Institutionen im gesellschaftlichen Umbruch, Opladen, 358-385.

Maus, Ingeborg 1994: Volkssouveränität vs. Konstitutionalismus. Zum Begriff der demokratischen Verfassung, in: Frankenberg, Günter (Hrsg.): Auf der Suche nach der gerechten Gesellschaft, Frankfurt a. M., 74-83.

Mayr, Ernst 1992: Speciational Evolution or Punctuated Equilibria, in: http://www.stephenjaygould.org/library/mayr_punctuated.html; 21.9.2010.

Meyer, John W. 1997: World Society and the Nation-State, in: American Journal of Sociology 103: 1, 144-181.

Meyer, John W. 2005: Weltkultur, Frankfurt a. M.

Möllers, Christoph 2003: Verfassungsgebende Gewalt - Verfassung - Konstitutionalisierung. Begriffe der Verfassung in Europa, in: von Bogdandy, Armin (Hrsg.): Europäisches Verfassungsrecht: Theoretische und dogmatische Grundzüge, Heidelberg, 1-57.

Moore, Barrington 1978: Injustice: The Social Bases of Obedience and Revolt, New York, NY. Moore, Robert I. 2001: Die Erste Europäische Revolution, München.

Morgenthau, Hans J. 1948: Politics Among Nations: The Struggle For Power and Peace, New York, NY.

Mosler, Hermann 1980: The International Society as a Legal Community, Alphen.

Neves, Marcelo 1992: Verfassung und positives Recht in der peripheren Moderne, Berlin.

Neves, Marcelo 1999: Zwischen Subintegration und Überintegration: Bürgerrechte nicht ernstgenommen, in: Kritische Justiz 32: 4, 557-577. 
Niesen, Peter 2010: Internationale Politische Theorie: Eine disziplinengeschichtliche Einordnung, in: Zeitschrift für Internationale Beziehungen 17: 2, 267-277.

O'Connor, James 1973: The Fiscal Crisis of the State. New York, NY.

Parsons, Talcott 1961: Order and Community in the International Social System, in Rosenau, James N. (Hrsg.): International Politics and Foreign Policy, New York, NY, 120-129.

Parsons, Talcott 1937: The Structure of Social Action: A Study in Social Theory with Special Reference to a Group of Recent European Writers. New York, NY.

Peters, Anne 2006: Compensatory Constitutionalism: The Function and Potential of Fundamental International Norms and Structures, in: Leiden Journal of International Law 19: 3, 579-610.

Piaget, Jean 1973: Das moralische Urteil beim Kinde, Frankfurt a. M.

Prien, Thore 2010: Fragmentierte Volkssouveränität - Recht, Gerechtigkeit und der demokratische Einspruch in der Weltgesellschaft, Baden-Baden.

Rancière, Jacques 2002: Das Unvernehmen. Politik und Philosophie, Frankfurt a. M.

Rawls, John 1971: A Theory of Justice, Cambridge, MA.

Rawls, John 1999: The Law of the Peoples, Cambridge MA.

Rosenthal, Joel 1991: Righteous Realists, Baton Rouge, LA.

Scheuerman, William E. 2009: Hans Morgenthau: Realism and Beyond, Cambridge.

Schmitt, Carl 1933: Völkerrechtliche Großraumordnung und Interventionsverbot für raumfremde Mächte, Berlin.

Schnädelbach, Herbert 2000: Hegels praktische Philosophie, Frankfurt a. M.

Sellin, Volker 2001: Die geraubte Revolution. Der Sturz Napoleons und die Restauration in Europa, Göttingen.

Shaw, Martin 2000: Theory of the Global State: Globality as Unfinished Revolution, Cambridge.

Skinner, Quentin 1989: Ambrogio Lorenzetti: The Artist as Political Philosopher, in: Belting, Hans/Blume, Dieter (Hrsg.): Malerei und Stadtkultur in der Dantezeit, München, 85-105.

Staff, Ilse/Müller, Christoph (Hrsg.) 1985: Staatslehre in der Weimarer Republik, Frankfurt a. M.

Stichweh, Rudolf 2000: Die Weltgesellschaft, Frankfurt a. M.

Streeck, Wolfgang 2010: Noch so ein Sieg, und wir sind verloren. Der Nationalstaat nach der Finanzkrise, in: Leviathan 38: 2, 159-173.

ten Brink, Tobias 2008: Geopolitik - Geschichte und Gegenwart kapitalistischer Staatenkonkurrenz, Münster.

Tilly, Charles 1990: Coercion, Capital, and European States, AD 990-1990, Cambridge, MA.

Tilly, Charles 1995: European Revolutions 1492-1992, Oxford.

Tomuschat, Christian 1995: Die internationale Gemeinschaft, in: Archiv des Völkerrechts 33, $1-20$.

Tugendhat, Ernst 2001: Moral in evolutionstheoretischer Sicht, in: Tugendhat, Ernst: Aufsätze 1992-2000, Frankfurt a. M., 199-224.

Tully, James 2002: The Unfreedom of the Moderns in Comparison to Their Ideals of Constitutionalism and Democracy, in: Modern Law Review 65: 2, 204-228.

von Bogdand,y Arnim/Venzke, Ingo 2009: In wessen Namen? Die internationale Gerichtsbarkeit diskurstheoretisch betrachtet, unv. Ms.

Vorländer, Karl 1921: Kants Leben, Leipzig.

Waschkuhn, Arno/Thumfart, Alexander (Hrsg.) 2002: Politisch-kulturelle Zugänge zur Weimarer Staatsdiskussion, Baden-Baden.

Weber, Max 1978: Gesammelte Aufsätze zur Religionssoziologie I, Tübingen.

Wiener, Antje 2008: The Invisible Constitution of Politics: Contested Norms and International Encounters, Cambridge.

Wiener, Antje 2010: Zur normativen Wende in den IB: Triangulation of a Different Kind, in: Zeitschrift für Internationale Beziehungen 17: 2, 335-354.

Wingert, Lutz 1993: Gemeinsinn und Moral, Frankfurt a. M. 
Witte, John 2002: Law and Protestantism: The Legal Teachings of the Lutheran Reformation, Cambridge.

Žižek, Slavoj 2001: Die Tücke des Subjekts, Frankfurt a. M. 